\title{
Sedation using 5\% lidocaine patches, midazolam and propofol in a combative, obese adolescent with severe autistic disorder undergoing brain magnetic resonance imaging
} -a case report-

\author{
Kwon Hui Seo ${ }^{1}$, Hong Soo Jung ${ }^{1}$, Eu Gene Kang ${ }^{1}$, Change Jae Kim ${ }^{2}$, Ho Young Rhee ${ }^{1}$, and \\ Yeon Soo Jeon ${ }^{1}$
}

Department of Anesthesiology and Pain Medicine, ${ }^{1}$ St. Vincent's Hospital, The Catholic University of Korea College of Medicine, Suwon, ${ }^{2}$ St. Paul's Hospital, The Catholic University of Korea College of Medicine, Seoul, Korea

\begin{abstract}
We present a 17-year-old man who underwent brain magnetic resonance imaging and laboratory exams for uncontrolled seizure. Patients with an autistic disorder require deep sedation or, occasionally, general anesthesia even for radiologic exams or simple procedures. The anesthetic management of an obese, violent patient with a severe autistic disorder and mental retardation can be challenging to anesthesiologists and requires a more careful approach in selecting adequate anesthetics and doses. This case emphasizes the importance of having a detailed plan to ensure the smooth process of premedication, anesthetic induction, maintenance, emergence and safe discharge of incorporated patients in the event of unexpected situations. A 5\% lidocaine patch to relieve the pain from the intramuscular injection and intravenous cannulation, intramuscular midazolam as premedication, and propofol for the maintenance of sedation can be a good sedation protocol in incorporated patients. (Korean J Anesthesiol 2014; 67: 421-424)
\end{abstract}

Key Words: Autistic disorder, Deep sedation, Magnetic resonance imaging, Midazolam, Obesity.

Received: June 30, 2014. Revised: August 5, 2014. Accepted: August 18, 2014.

Corresponding author: Yeon Soo Jeon, M.D, Ph.D., Department of Anesthesiology and Pain Medicine, St. Vincent's Hospital, The Catholic University of Korea College of Medicine, 93, Jungbu-daero, Paldal-gu, Suwon 442-723, Korea. Tel: 82-31-249-7274, Fax: 82-31-258-4212, E-mail: likewinds@catholic.ac.kr

(c) This is an open-access article distributed under the terms of the Creative Commons Attribution Non-Commercial License (http:// creativecommons.org/licenses/by-nc/3.0/), which permits unrestricted non-commercial use, distribution, and reproduction in any medium, provided the original work is properly cited. 
Autism is a common disorder characterized by impairments in social interaction, communication, and developmentally inappropriate behavior, interests, or activities. Children with autism display an apparently inflexible adherence to specific, nonfunctional routines or rituals [1]. When placed in an unfamiliar environment or confronted with strangers, they become more strained, make attempts to break away, or exhibit violent behavior. These characteristics make it difficult for physicians to perform appropriate exams and treatments. Therefore, autistic children need to be deeply sedated for radiologic examination or simple procedures and sometimes general anesthesia is imperative. Anesthetic plans for such patients should include prudent preparations for a variety of situations, and adaptable guidelines [2].

In particular, autistic children with other comorbid conditions such as neurologic or medical problems may demand more careful approach for selecting adequate anesthetics and doses. They are more vulnerable to the adverse effects of sedation and of anesthesia outside the operating room. There are very few reports about autistic patients with morbid obesity and uncontrolled epileptic seizures. We report the anesthetic management of an obese patient with an autistic disorder, moderate mental retardation, and uncontrolled epileptic seizure undergoing brain magnetic resonance imaging (MRI).

\section{Case Report}

A 17-year-old male with a height of $174 \mathrm{~cm}$ and a weight of $100 \mathrm{~kg}$ (body mass index 33.0) was scheduled for a brain MRI and laboratory exams under deep sedation. The patient had been diagnosed with severe autism at the age of 4 and moderate mental retardation (intelligence quotient of 41) at the age of 7 . He had not been able to receive any vaccinations or physical exams since the age of 7 due to combative behavior in unfamiliar surroundings and in the presence of strangers. His pediatrician had been always unable to examine him in the outpatient clinic and no blood test had been performed even once. Two years prior, he had suffered from a first attack of epileptic seizure and had started taking topiramate $200 \mathrm{mg}$ bid. Nevertheless, the seizure was poorly controlled, and the topiramate dosage was increased to $400 \mathrm{mg}$ bid. The pediatrician recommended laboratory exams to check the plasma concentration of the drug and a brain MRI. Sixteen months prior, the patient was consulted for sedation for the brain MRI. We believed that an intravenous (IV) cannulation for propofol sedation would be possible after ingestion of oral chloral hydrate. However, the patient was severely agitated and ran out of the sedation room. He ingested only $1 \mathrm{~g}$ $(10 \mathrm{ml})$ of chloral hydrate on a bench. We tried to perform the IV line when he became a little quieter. He severely struggled and pushed his parents and the physician away. We could not prevent the violent resistance of a big child, and our 2 hour long first attempt failed. Two months ago, the patient began to show more violent and aberrant behavior and a generalized tonic clonic type of seizure started to appear. Levetiracem 1,000 $\mathrm{mg}$ bid were added to his drug regimen but, even under a high dose of medication, the seizure was not controlled. He was reconsulted for the sedation.

The day before the MRI, we explained the necessity of physical restraint, forcible intramuscular (IM) injection, and the possibility of intubation to the patient's parents. We got informed consent and prescribed 5\% lidocaine patches. We taught his parents to attach the small lidocaine patch cuts to his deltoid muscles and medial wrists after he fell asleep the previous night.

On the day of the MRI, his parents gave him a toilet kit on the bench in the corridor. We prepared midazolam $15 \mathrm{mg}(3 \mathrm{ml})$ with a 23 gauge needle, we could inject only midazolam 12.5 mg into patient's left deltoid muscle due to severe his struggle despite the help of 6 people ready to restraint. He became sleepy in about 10 minutes and his mother instructed him to lay on the gurney. After checking his respiration and radial pulse, we moved him to the sedation room. Upon the arrival in the sedation room, he was deeply asleep, and peripheral oxygen saturation $\left(\mathrm{SpO}_{2}\right) 91 \%$, heart rate (HR) 85 beats/min and assured blood pressure (BP) over $80 / 60 \mathrm{mmHg}$ by palpation of the radial pulse. With administration of oxygen $2 \mathrm{~L} / \mathrm{min}$ through a nasal prong, $\mathrm{SpO}_{2}$ was maintained above $95 \%$. We cannulated a 20 gauge angiocatheter into a peripheral vein of his left wrist, sampled $10 \mathrm{ml}$ of blood and injected glycopyrrolate $0.2 \mathrm{mg}$ intravenously to avoid bradycardia by propofol and to reduce oral secretion. Thereafter, we moved the patient to the MRI suite maintaining the portable monitor. It took about 30 minutes after the IM injection of midazolam until he arrived onto the scan desk in the MRI suite and there was no resistance or movement of the patient. We changed the oxygen supply and fitted an MRIcompatible monitor showing $\mathrm{SpO}_{2} 95 \%$ and $\mathrm{HR} 81$ beats/min. Because of no room for the head set by his very large head size, we put ear plugs on his ears and fixed his folded ears to his lateral cheeks with plasters. This process awakened him and he began to struggle. We administered him $1 \%$ propofol $120 \mathrm{mg}$ intravenously. After he fell asleep again, $\mathrm{SpO}_{2}$ was checked as $85 \%$, and we inserted an oral airway and performed jaw thrust maneuver. After $\mathrm{SpO}_{2}$ was raised to $94 \%$, we connected $0.2 \%$ propofol mixed with normal saline $200 \mathrm{ml}$ to the side of the main fluid and infused it at a velocity of $6 \mathrm{mg} / \mathrm{kg} / \mathrm{hr}$ via an MRI-compatible dropper (MRidium ${ }^{\mathrm{TM}} 3850$ MRI infusion system, IRadimed Corporation, FL, USA). We checked his BP 110/76 mmHg, HR 77 beats/min, confirmed no movement, and the MRI was initiated. We watched the patient's movement and chest excursions through the camera. $\mathrm{SpO}_{2} 95-96 \%$ and $\mathrm{HR} 78-86$ beats/min were maintained, and the $\mathrm{BP}$ was recorded as $102 / 65 \mathrm{mmHg}$ and $91 / 62 \mathrm{mmHg}$ at 20 minutes and 35 minutes respectively 
after the start of exam. The MRI was completed about 52 minutes later. A propofol mixed fluid was injected continually at the same speed to prevent awakening until the patient was moved on to the gurney. The total dose of used propofol, including a bolus dose, was about $620 \mathrm{mg}$. When we arrived in the sedation room, BP 112/58 mmHg, HR 82 beats/min, and $\mathrm{SpO}_{2} 96 \%$ were checked. About 10 minutes after (110 minutes after midazolam injection), he showed a lethargic response to light shaking. We removed the venous cannulation due to worries about his violent behavior. He opened his eyes spontaneously about $45 \mathrm{~min}$ utes after returning to the sedation room. There were no adverse effects such as paradoxical emergence agitation or nausea and vomiting. He then walked out of the sedation room by himself. He started the arrangement of his toilet kit as usual on the bench of the corridor. He was discharged home about 1 hour and 20 minutes after the end of the MRI.

\section{Discussion}

We confirmed that $5 \%$ lidocaine patches, IM midazolam and propofol can be a good sedation protocol for performing a brain MRI on a violent, obese patient with a severely autistic and epileptic disorder. Associated central nervous system involvement is very common in autistic patients, and up to $30 \%$ of those patients suffer from some type of seizure disorder [3]. Several reports have suggested an appropriate anesthetic management protocol for radiologic exams in such patients $[2,4,5]$, which can be divided into three stages; premedication, anesthesia induction and maintenance, and emergence for discharge.

First, due to the noncooperation of autistic patients, premedication is an essential stage for IV cannulation in order to make the smooth induction and maintenance of anesthesia possible. Anesthesiologists should consider concurrent diseases and drug interactions with induction agents in their choice of premedication drugs. Because most patients do not require admission, the onset and duration of the agents should be accurately estimated. Most previous studies have recommended oral agents like oral midazolam or ketamine for premedication. However, discharge of those patients was delayed $[2,4,5]$.

IM injection in autistic patients always requires physical restraints due to the pain caused by the injection. Patients become more anxious and violent, can provoke the injection of an insufficient dose of anesthetic agents, the break of the angioneedle, needle stick injuries, or other physical injuries to the hospital staff. Therefore, we should consider interventions like basic explanation or teaching, a theater visit, play therapy, mock inductions, reward, premedication and family counseling [6]. Kim et al. [7] insisted that pretreatment with a 5\% lidocaine patch was effective in reducing the pain during venipuncture in pediatric patients. Gammaitoni et al. [8] reported that there were little side effects and drug interactions after testing with four 5\% lidocaine patches for 3 consecutive days in healthy volunteers. In our case, ketamine was contraindicated as the patient had active seizures. Admission was also impossible and general anesthesia was not mandatory. Because we needed a rapid, definite onset and recovery in the setting of the outpatient clinic, we selected IM midazolam. We used 5\% lidocaine patches to relieve the pain from the IM injection, and used his favorite objects to divert his attention. The lidocaine patches could not prevent the patient's resistance entirely, but our trial was somewhat successful.

Because this patient was diagnosed with class I obesity and had a failed history of using oral sedatives, we decided to administer the induction dose of midazolamin order to avoid the use of multiple drugs. In obese subjects, the total volume of distribution is increased about three times over that in nonobese subjects, with no difference in the clearance rates. Thus, a single induction dose of midazolam needed to be administered based on the total body weight [9]. A high dose of bolus midazolam and concomitant use of gamma-aminobutyric acid agonists as antiepileptics can cause respiratory depression. Midazolam also has other shortcomings such as paradoxical disinhibition which can occur at any dose and dysphoria in higher doses. If so, IV or transmucosal routes flumazenil can be administered [10]. Nevertheless, IM high dose of midazolam has been used for sedation of combative or epileptic patients in the emergency departments [11]. We thought it would be safer if an intubation set, oxygen, flumazenil with trained anesthesiologist are prepared.

Second, the induction and maintenance anesthetic agent chosen for sedation in the MRI suite was propofol. Propofol has been considered as a perfect drug for sedation because it can be titrated effectively to the appropriate sedation level and presents a short recovery time [12]. General anesthesia during MRI may be safer in terms of airway management and easier for controlling the depth of anesthesia in obese patients, but, it presents many disadvantages including intubation, ventilator weaning, and rigorous monitoring. Besides, for patients with large heads as in our case, there may be no room for the endotracheal tube to be placed or kinked within the surface coil. Therefore, we selected non-intubated IV sedation with propofol rather than general anesthesia. The MRI suite needs a long-distance ventilator and capnogram, special electrocardiography, a blood pressure manometer, and an oximeter to avoid the effects of the magnetic field. Nevertheless, even occasional blood pressure measurements may cause a lowering of the image quality clinically. To our regret, we did not monitor the capnography even if it is indispensable for sedation in the MRI suite, but we could assure the chest excursion through a camera placed inside the MRI suite.

Generally, antiepileptic drugs may have additive sedative effects with anesthetic drug and enzyme induction by antiepileptic drugs may alter the pharmacokinetics and pharmacodynamics 
of anesthetic drugs [13]. However, there have been no papers that clearly reported about the changes of anesthetic requirement by two antiepileptic drugs used in our case. We administered propofol $120 \mathrm{mg}(1.2 \mathrm{mg} / \mathrm{kg})$ for induction and about 6 $\mathrm{mg} / \mathrm{kg} / \mathrm{hr}$ for maintenance of the anesthesia. Because both the volume of distribution and clearance of propofol increase in an obese patient, both the induction and maintenance dose for an obese patient should be based on the total body weight [9]. Machata et al. [14] proposed that the appropriate dose of propofol for pediatric patients undergoing a MRI is $2-5 \mathrm{mg} / \mathrm{kg} / \mathrm{hr}$ and excessive doses of propofol may result in unintended deep sedation and respiratory depression. Asahi et al. [15] proved that 7 to 19 year old autistic patients were required about propofol 10 $\mathrm{mg} / \mathrm{kg} / \mathrm{hr}$ during dental treatment under non-intubated IV general anesthesia. They concluded that autistic patients may have a greater requirement for propofol because of the increased serum levels of glutamate and hypersensitivity to acoustic stimuli. In our case, although we administered a decreased induction dose of propofol considering the residual effect of midazolam, desaturation $\left(\mathrm{SpO}_{2} 85 \%\right)$ occurred. In the MRI suite, intubation using laryngoscope is impossible, so, we prepared ambu bag with mask and laryngeal mask airway and $\mathrm{SpO}_{2}$ rose immediately in response to head tilting and forward jaw thrusting. The maintenance dose of $6 \mathrm{mg} / \mathrm{kg} / \mathrm{hr}$ did not lead to desaturation, hypotension, or bradycardia. Furthermore, there was no need for an additional bolus dose.

Third, many studies have pointed out the difficulties in the recovery and admission care of autistic patients [2,6]. After exams and procedures, the ultimate aim is to safely discharge the patients as early as possible so as to return them to an everyday environment [6]. In our case, the patient recovered a presedative consciousness level within about one hour after the MRI. The administration of midazolam premedication through an IM rather than an oral route and the immediate clearance of propofol may have contributed to the rapid recovery. Therefore, this constituted an appropriate management protocol for deep sedationin an outpatient clinic.

In conclusion, there should be several clinical deliberations about the various onsets of sedatives, inadequate sedation, sedation failure or adverse effects by oversedation at each stage. A prolonged induction time, repeated MRI sequences, or rescheduling because of failed sedation are costly with respect to increased personnel time and the down time of the MRI scanner. Not only such factors but also delayed recovery causes inconvenience to both patients and the hospital staff. Therefore, in incorporated patients such as an autistic disorder, we should perform sedation for radiologic exams following a detailed, stageby-stage plan designed for a range of unexpected situations.

\section{References}

1. Raviola G, Gosselin GJ, Walter HJ, DeMaso DR. Pervasive developmental disorders and childhood psychosis. In: Nelson Textbook of Pediatrics. 19th ed. Edited by Kliegman RM, Stanton BF, St. Geme JW, Schor NF, Behrman RE: Philadelphia, WB Saunders Company. 2011, pp 100-7.

2. van der Walt JH, Moran C. An audit of perioperative management of autistic children. Paediatr Anaesth 2001; 11: 401-8.

3. Volkmar FR, Nelson DS. Seizure disorders in autism. J Am Acad Child Adolesc Psychiatry 1990; 29: 127-9.

4. Shan S, Shah S, Apuya J, Gopalakrishnan S, Martin T. Combination of oral ketamine and midazolam as a premedication for a severely autistic and combative patient. J Anesth 2009; 23: 126-8.

5. Rainey L, van der Walt JH. The anaesthetic management of autistic children. Anaesth Intensive Care 1998; 26: 682-6.

6. Christiansen E, Chambers N. Induction of anesthesia in a combative child; management and issues. Paediatr Anaesth 2005; 15: 421-5.

7. Kim CH, Yoon JU, Lee HJ, Shin SW, Yoon JY, Byeon GJ. Availability of a $5 \%$ lidocaine patch used prophylactically for venipuncture- or injection-related pain in children. J Anesth 2012; 26: 552-5.

8. Gammaitoni AR, Alvarez NA, Galer BS. Safety and tolerability of the lidocaine patch 5\%, a targeted peripheral analgesic: a review of the literature. J Clin Pharmacol 2003; 43: 111-7.

9. Ogunnaike BO, Whitten CW. Evaluation of the obese patient. In: Anesthesiology. 2nd ed. New York, McGraw-Hill. 2012, pp 308-10.

10. Kanegaye JT, Favela JL, Acosta M, Bank DE. High-dose rectal midazolam for pediatric procedures: a randomized trial of sedative efficacy and agitation. Pediatr Emerg Care 2003; 19: 329-36.

11. Isbister GK, Calver LA, Page CB, Stokes B, Bryant JL, Downes MA. Randomized controlled trial of intramuscular droperidol versus midazolam for violence and acute behavioral disturbance: the DORM study. Ann Emerg Med 2010; 56: 392-401.

12. Schulte-Uentrop L, Goepfert MS. Anaesthesia or sedation for MRI in children. Curr Opin Anaesthesiol 2010; 23: 513-7.

13. Pasternak JJ, Lanier WL Jr. Disease affecting the brain. In: Stoelting's Anesthesia and Co-Existing Disease. 6th ed. Edited by Hines RL, Marshall KE: Philadelphia, Saunders Elsevier. 2012, pp 218-54.

14. Machata AM, Willschke H, Kabon B, Kettner SC, Marhofer P. Propofol-based sedation regimen for infants and children undergoing ambulatory magnetic resonance imaging. Br J Anaesth 2008; 101: 239-43.

15. Asahi Y, Kubota K, Omichi S. Dose requirements for propofol anaesthesia for dental treatment for autistic patients compared with intellectually impaired patients. Anaesth Intensive Care 2009; 37: 70-3. 